\title{
Strain-based tunable optical microresonator with an in-fiber rectangular air bubble
}

\author{
Shen liu ${ }^{1,2}$, Zhongyuan Sun ${ }^{1,2}$, Lin Zhang ${ }^{2}$, Cailing Fu ${ }^{1}$, Yu liu ${ }^{3, *}$, Changrui \\ LIAO $^{1}$, JUN HE ${ }^{1}$, ZHIYONG BAI ${ }^{1}$, YING WANG ${ }^{1}$ AND YIPING WANG ${ }^{1, *}$
}

\author{
${ }^{1}$ Key Laboratory of Optoelectronic Devices and Systems of Ministry of Education and Guangdong Province, \\ College of Optoelectronic Engineering, Shenzhen University, Shenzhen 518060, China \\ ${ }^{2}$ Aston Institute of Photonic Technologies, Aston University, Birmingham, UK \\ ${ }^{3}$ College of Optoelectronic Engineering, Chongqing University of Posts and Telecommunications, Chongqing, China
}

*Corresponding author:ypwang@szu.edu.cn; liuyu@cqupt.edu.cn

Received XX Month XXXX; revised XX Month, XXXX; accepted XX Month XXXX; posted XX Month XXXX (Doc. ID XXXXX); published XX Month XXXX

We demonstrate a strain-based fully tunable, nearlossless, whispering-gallery-modes (WGMs) resonator made of an in-fiber rectangular air bubble (RAB), which is fabricated by splicing two segments of standard single mode fibers. Such a resonator, with a $39 \mu \mathrm{m}$ order radius and $1 \mu \mathrm{m}$ order wall thickness, contributes to a high quality factor exceeding $\mathbf{1 0}^{6}$. The tuning in resonant wavelength is achieved via applying tensile strain to the resonator and the voltage-tuning rate of WGM resonance peaks is about $31.96 \mathrm{pm} / \mathrm{V}$ (strain-tuning rate $\sim 14.12$ $\mathrm{pm} / \mu \varepsilon$ ), and the corresponding tuning accuracy is better than $0.03 \mathrm{pm}$. Since the tensile strain applied on the resonator can reach $1000 \mu \varepsilon$, the achievable total tunable bandwidth of $\mathbf{1 4 . 1 2} \mathbf{n m}$ is more than two times of its azimuthal free spectral range.(C) 2018 Optical Society of America

OCIS codes: Resonators (230.5750), Fiber optics sensors (060.2370),

Microstructure fabrication (220.4000), Microstructured fibers (060.4005), whispering-gallery-modes.

http://dx.doi.org/10.1364/OL.99.099999

Optical whispering gallery-modes (WGMs) resonators, with a combination of high quality factor (Q) and small mode volume of modes, have great potential of growth in many research and technology fields [1], such as microlasers [2-4], biosensors [5,6], chemical sensing [7], and interface for quantum communication[8,9]. A variety of resonators are currently demonstrated, covering the structures of microsphere [10], microtoroid [6], microring[11], and mirobubble[12]. Those resonators typically confine the light in a narrow ring along its equator surface by continuous total internal reflection. While such equatorial WGMs have the advantage of a small mode volume they also exhibit a large wavelength spacing between consecutive modes [13]. Furthermore, for many of applications, matching of a resonant mode to a narrow wavelength range is required. One of the direct methods to preselect the WGM resonant wavelength is by means of the size design of the resonator. However, the fabrication limitations hinder precise control over mode peak position. Another method is to directly tune the already generated resonant mode. Therefore, various methods to tune the resonator have been reported currently, examples including applying strain [13-15], pressure [15,16], electric-field [17,18], magnetic-field [19], and bending [20].

In this letter, for the first time to the authors' knowledge, we propose and experimentally demonstrate a novel high- $Q$ microresonator based on an in-fiber rectangular air bubble (RAB) structure, and its WGMs resonance tuneability by applying tensile strain. Such a microresonator, with a diameter of $78 \mu \mathrm{m}$ along its equator surface and an ultrathin wall thickness of $1 \mu \mathrm{m}$, is created by splicing two sections of standard single mode fibers [21]. We have successfully demonstrated the possibility of a reliable continuous tuning of WGMs in the resonator, by simply applying the tensile strain to the structure and a tunability of $31.96 \mathrm{pm} / \mathrm{V}$ is achieved, giving a tunable bandwidth of $14.12 \mathrm{~nm}$. The microresonator has also exhibited a superbroad tunable range, which is over two times larger than of its azimuthal free spectral range (FSR). In our experiment, the achieved quality factor of the resonator exceeds $10^{6}$ and the WGM is recognized as near-lossless.

As shown in Fig.1(a), an RAB, as a resonator, has a diameter $\left(2 \mathrm{R}_{0}\right)$ of $78 \mu \mathrm{m}$ along its equator surface and a maximum cavity length of $85 \mu \mathrm{m}$ along the fiber axis that have been measured from optical microscope image. This RAB is fabricated by means of splicing two sections of standard single mode fiber together and tapering the splicing joint, and the detailed fabrication process is described in Ref. [21, 22]. It is noteworthy that in the whole fabrication process of the RAB, only a common fusion splicer was employed, and no additional device required like in using CO2 laser beam, pressurization or convective heating in a flame and furnace would be involved $[12,14]$. To determine the fabrication limit of the silica diaphragm thickness, the RAB was cut open at the A-point labeled on the equator surface (Fig.1(a)) by using femtosecond laser micromachining. A scanning electron microscope (SEM) image shown in inset of Fig.1(a), gives a measured wall thickness of the RAB to be about $1 \mu \mathrm{m}$. Such an RAB, with an ultrathin wall thickness, has excellent optical and mechanical properties. We have investigated the WGMs of the RAB microresonator by coupling WGMs through a taper fiber, as 


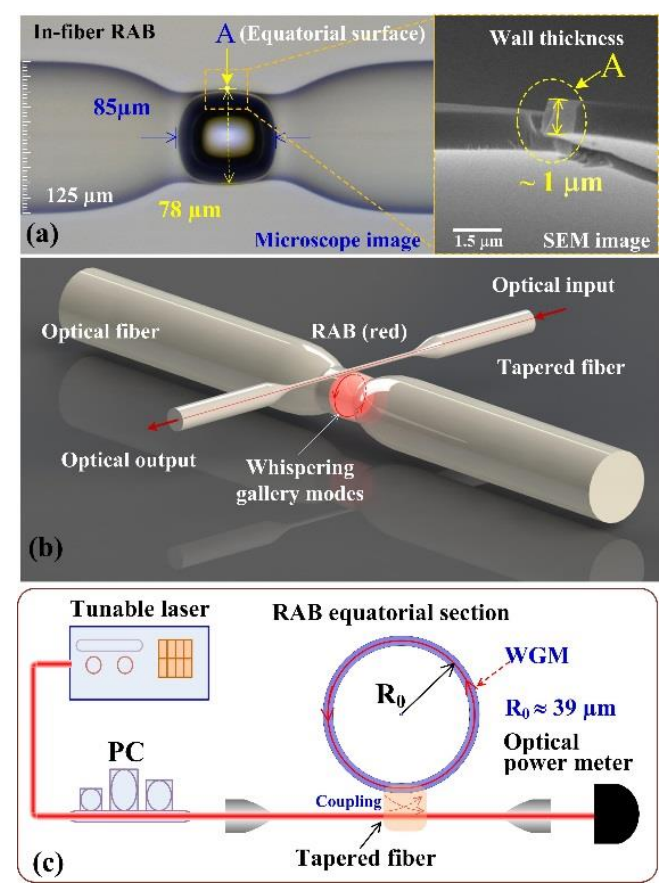

Fig.1. (a) The optical microscope image of an in-fiber RAB, and the inset, the cut-plane SEM image of the RAB. (b) Schematic diagram of a fibertaper coupled micro-resonator. (c) Schematic of the experimental setup; PC, polarization controller.

shown in Fig.1 (b). The taper-fiber, with a biconical taper and a symmetrical waist, was fabricated from a conventional single-mode fiber by means of the oxyhydrogen flame direct heating method. In the experiment, the taper-fiber waist, with a diameter of $\sim 1 \mu \mathrm{m}$ and its near zero transmission loss character, was positioned in direct contact with the in-fiber RAB along its equator surface, as illustrated in Fig. 1(b). The light can be coupled into the RAB resonator through this taper-fiber, which evanescently excites the WGMs in the RAB along its equator, and the coupling strength can be adjusted by changing the air gap between the taper-fiber and the equator surface of RAB. The WGM of RAB resonator can be measured using the set-up shown in Fig,1(c), where the input end of the taper-fiber is connected to a tunable laser source (Agilent, 81940A) via a polarization controller, and the other end is to an optical power meter (Agilent, N7744A).

As shown in Fig.2, the equatorial WGMs of the RAB resonator were measured by the optical power meter with a resolution of 0.6 pm covering the wavelength range from 1550 to $1585 \mathrm{~nm}$. While such equatorial WGMs have a small mode volume, they also exhibit a large azimuthal FSR between consecutive modes, and the measured azimuthal FSR is about $6.9 \mathrm{~nm}$ around the wavelength of $1556 \mathrm{~nm}$. Here, the azimuthal FSR could be expressed as [14]

$$
\Delta \lambda_{F S R}^{(a z i m)}=\lambda^{2} /\left(2 \pi n_{e f f} R_{0}\right)
$$

where, $\lambda$ is the resonant wavelength, $n_{\text {eff }}$ is the effective index of the silica, and $\mathrm{R}_{0}$ is radius of the rectangular air bubble along the equator surface. As shown in Fig.2, the wavelengths of WGM resonant peaks are about 1553.219, 1560.0848, 1567.0118, 1573.9976, and $1581.0428 \mathrm{~nm}$, respectively. Based on Eq. (1), the calculated $\mathrm{R}_{0}$ is about $38.9 \mu \mathrm{m}$, which agrees well with the measurement result from the optical microscope image (Fig.1(a)).

Furthermore, as shown in the inset of Fig.2, the estimated resonance $Q$-factor obtained by Lorentzian fitting is of $\sim 1.23 \times 10^{6}$.

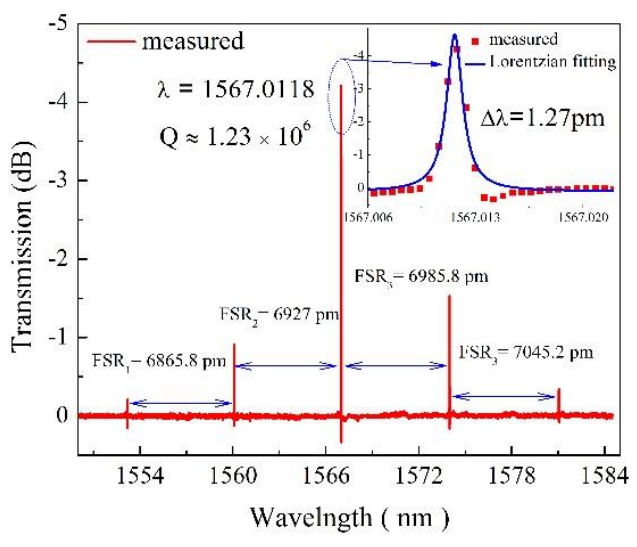

Fig. 2. The measured WGM transmission spectra with a wavelength range of 1550 to $1584 \mathrm{~nm}$. Inset: magnified measurement data near a resonant peak (red-dots) fitted with a Lorentzian curve (blue-line)

In addition, it also can be seen that the transmission spectrum of the $\mathrm{RAB}$ resonator is near-lossless as the measured loss is only about $0.03 \mathrm{~dB}$, which owing to the factor of a very smooth inner and outer surface of the RAB ensuring better confinement of the light in a narrow ring along the equator surface by continuous total internal reflection. Note, the positive dips occurred under 0 -dB level are due to the measurement error caused by the power meter, thus not real signal.

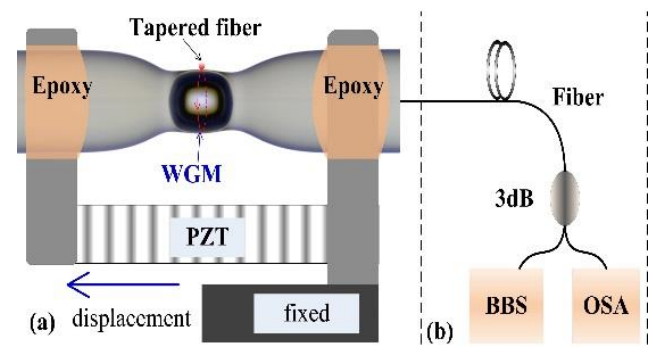

Fig.3. (a) Illustration of experimental set-up used for applying the tensile strain to the resonator; (b) The set-up used for real-time monitoring the spectral changes of RAB Fabry-Perot interferometer.

According to Eq.(1), it can be seen that any FSR interval of length $\Delta \lambda_{F S R}^{(\text {amiz) }}$ contains a full set of resonances, which correspond to WGMs under different quantum numbers. For this reason, a resonator can be regarded as fully tunable if its tuning wavelength range exceeds the FSR, i.e. $\Delta \lambda_{F S R}^{(a m i z)}$ [14]. Thus, a resonance, corresponding to any transversal WGM distribution of a fully tunable resonator, can be shifted to any predetermined wavelength. To evaluate this tunable function, an experiment was set up to apply tensile strain to the $\mathrm{RAB}$ resonator for tuning the resonance wavelength of WGMs, as shown in Fig.3. To apply the tensile strain to resonator, one end of the RAB was glued on a fixed stage, and another end was fixed to a piezoelectric transducer (PZT) controlled stage. In order to measure the actual applied strain to the $\mathrm{RAB}$, a broadband light source (BBS), a 3-dB fiber coupler, and an optical spectrum analyzer (OSA) with a resolution $0.01 \mathrm{~nm}$ were employed to directly monitor the cavity length variation (i.e. applied tensile strains) of the RAB in real time, as shown in Fig.3(b). 

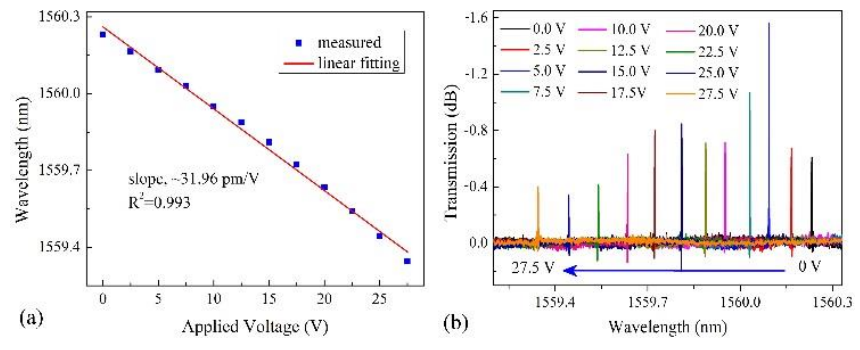

Fig.4. (a) Applied PZT voltage to resonator and the calculated voltagetuning rate of about $31.96 \mathrm{pm} / \mathrm{V}$. (b) Spectrum evolution of the resonator based on RAB with PZT voltage increases from 0 to $27.5 \mathrm{~V}$.
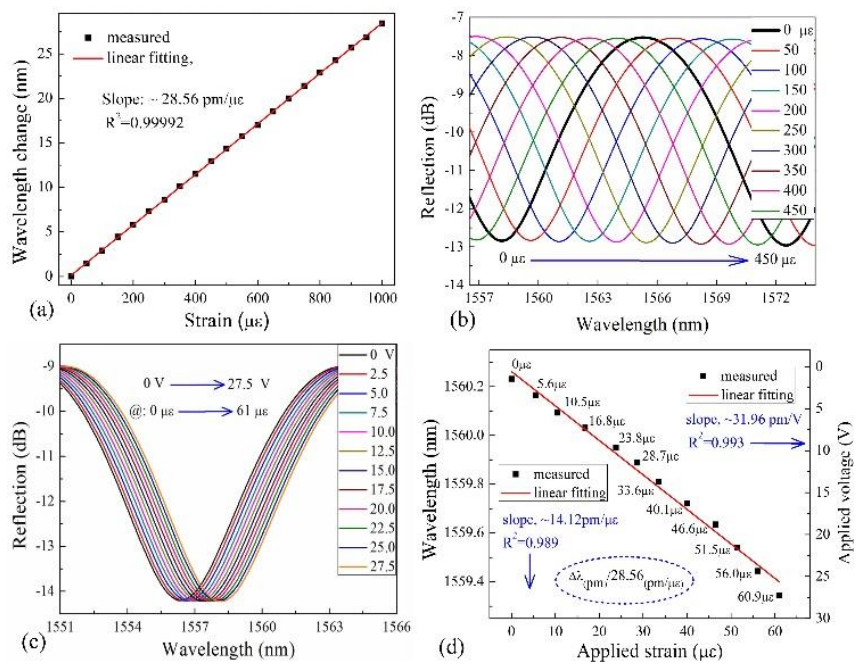

Fig.5. (a) Strain response and (b) spectral evolution of the RAB FPI under manual stretching. (c) Spectral evolution of RAB FPI under PZT applied voltage from 0 to $27.5 \mathrm{~V}$. (d) The correlation between then voltage-tuning rate and strain-tuning rate.

To investigate the strain-based tunability of the RAB resonator, the wavelength shift of the WGMs was measured, while the applying PZT voltage varied from 0 to $27.5 \mathrm{~V}$ in an increment of 2.5 V. The correlations plotted in Fig.4(a), and its slope of the linear fitting gives a tuning rate of about $31.96 \mathrm{pm} / \mathrm{V}$ with a correlation factor of $\mathrm{R}^{2} \sim 0.993$ showing a good linearity of wavelength change with increasing applied-voltages. The corresponding WGM resonance spectrum evolution, with increasing PZT voltage, is illustrated in Fig.4(b), measured by the optical power meter with a resolution of $0.15 \mathrm{pm}$. As the figure shows, the coupling intensity of the WGMs has shown a fluctuation (increase or decrease), which is attributed to the position change of the probing point between the taper-fiber and RAB due to the applied strain. The resonances dips shifted toward shorter wavelength, i.e. blue shift, which indicates that the equatorial radius $\mathrm{R}_{0}$ is reduced with the PZT voltage increasing. This correlation was also proved in the numerical simulation, as shown in Fig.6, where we clearly see that the radius $\mathrm{R}_{0}$ is also reduced with increased tensile strain applied to the RAB. Note that, here, the increased PZT voltage is equivalent to the tensile strain applied to the RAB. Furthermore, the employed PZT is the model of PI-888.91 with a sub-nanometer resolution (https://www.piceramic.com/en/), which can respond well to milli-voltage (mV), thus from the tuning rate of $31.96 \mathrm{pm} / \mathrm{V}$, we can estimate the tuning accuracy is approximately $0.03 \mathrm{pm}$.

In the experiment, due to the restriction from the extension length of the PZT, we were not able to measure the actual full tunable range of the resonator. To evaluate the full tunable range of $\mathrm{RAB}$ resonator, the stretching strength of the $\mathrm{RAB}$ was measured as a Fabry-Perot interferometer (FPI) by manually operating the translation stage by the set-up shown in Fig.3(b). Fig.5 (a) plots the FPI wavelength shift against the tensile strain, which was increased from 0 to $1000 \mu \varepsilon$ with a step of $50 \mu \varepsilon$ by manually stretching the RAB. From Fig.5(a), the strain sensitivity of the RAB FPI is calculated to be $28.56 \mathrm{pm} / \mu \varepsilon$, corresponding to a linear fitting with a correlation factor of $\mathrm{R}^{2}=0.99992$. Fig.5 (b) and (c) show the spectral evolution of the RAB FPI under manual and PZT (0 to $27.5 \mathrm{~V}$ ) stretching, respectively. From Fig.5 (a) and (c), we can build a correlation between the applied voltage and tensile strain, as plotted in Fig.5(d). Thus, we can see the applied PZT voltage from 0 to $27.5 \mathrm{~V}$ corresponds to the tensile strain from 0 to $60.9 \mu \varepsilon$. From Fig.5(d), we can obtain a strain-tuning WGM rate to be about 14.12

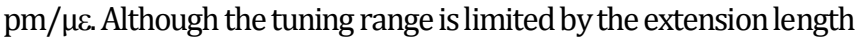
of the PZT, according to the measured results in Fig.5(a), the available stretch range of $1000 \mu \varepsilon$ will allow a total WGM tunable range of $\sim 14.12 \mathrm{~nm}$ for the RABresonator, which is two times more than its azimuthal FSR of $6.9 \mathrm{~nm}$. The tunability figure of merit of this RAB WGM is about $\Delta \lambda / \Delta \lambda_{F S R}^{(\text {amiz) }} \approx 2.05$.

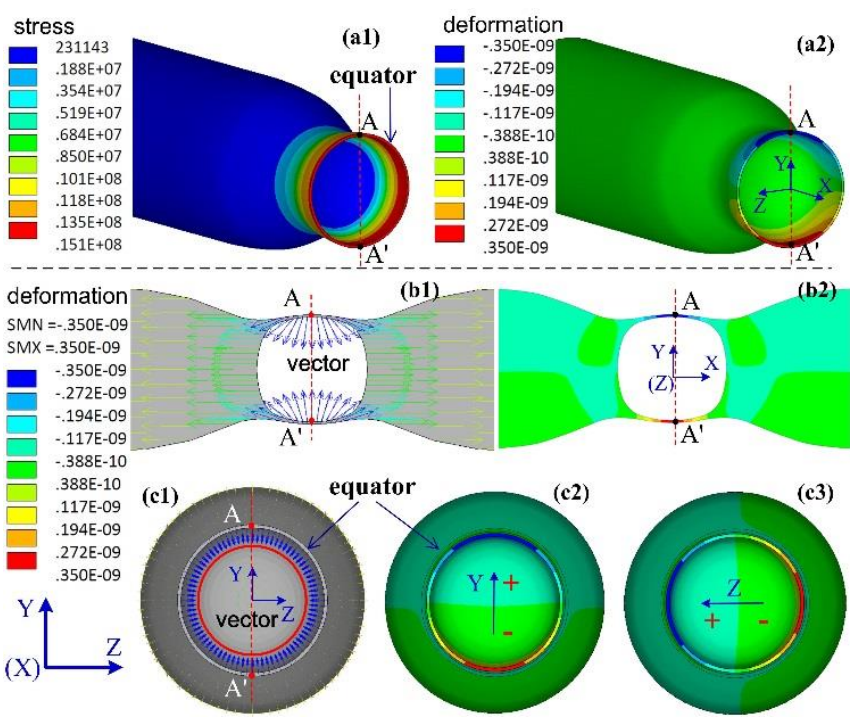

Fig.6.(a1) Three-dimensional stress distribution contours and (a2) the stress distribution contours along the $\mathrm{Y}$ direction. The deformation vector field of RAB along (b1) the transverse section and (c1) the longitudinal section. The corresponding deformation of the RAB under $1 \mu \varepsilon$ strain for (b2) the transverse section (Y-axis), (c2) longitudinal section(Y-axis) and (c3) longitudinal section (Z-axis), respectively.

In order to investigate the strain-based tuning mechanism of the WGM resonator, the structural deformation and stress distribution of the RAB under strain were calculated by establishing a simulation models utilizing commercial software of finite element analysis. Fig.6 (a1) illustrates the three dimensional stress distribution contour of the resonator, which is mirror model 


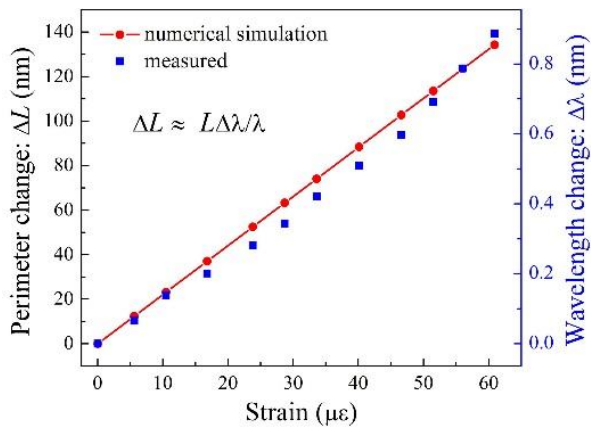

Fig. 7. The results of numerical simulation for the perimeter change (left axis) along the equator surface of the RAB versus the applied tensile strain (red dots), reflecting a corresponding wavelength change (right axis) of the WGMs in the optical micro-resonator based on RAB and comparison with the measured results (blue blocks).

along the equator of the $\mathrm{RAB}$, with a tensile strain of $1 \mu \varepsilon$, and the colors indicate the stress distribution in different parts of the resonator. It can be seen that the maximum stress is distributed on the equator surface of the resonator, so that the equator deformation is also maximum value, which is illustrated in different views, as shown in Fig.6 (a2), (b2), (c2), and (c3), respectively. The vector displacement contours are shown in Fig.6 (b1) and (c1), and the arrows describe the directions of the resonator's deformation. As the marked-points $\mathrm{A}$ and $\mathrm{A}^{\prime}$ at the equator surface of the resonator in Fig.6(a, b), it is clear that the marked-points shift toward the inner center of the resonator, indicating the equatorial radius $\mathrm{R}_{0}$ is reduced. The calculated results also show that the resonator's equator perimeter length, used to support the fundamental mode resonance, is gradually decreasing with the tensile strain increasing. According to Eq.(1), the perimeter length is $\mathrm{L}=2 \pi \mathrm{R}_{0}$, and the changing length $\Delta \mathrm{L}$ (red dots) is calculated by numerical simulation with the increased tensile strain, as shown in Fig.7 (left axis). Furthermore, the measured changing length (blue square) can be calculated by, $\Delta \mathrm{L}=\Delta \lambda(\mathrm{L} / \lambda)$, where the strain induced wavelength change $\Delta \lambda$ is measureable. From Fig.7, we can clearly see that the theoretical calculation of the perimeter length change agrees very well with the measured values. Comparing with other microresonators formed micro- bubble, toroid and ring, the $\mathrm{RAB}$ resonators clearly show several advantages, such as small volume, relative robustness and high- $\mathrm{Q}$ thus could be more suitable for specific applications, for example, the investigation of cavity quantum electrodynamics (QED), because there is a small bubble in the resonator that may be used to contain atoms [25]. Furthermore, the RAB resonator has a simple and low-cost fabrication process, as only by using a fusion splicer to form a smaller mode volume WGMs resonator.

In conclusion, we have successfully shown the implementation of a reliable continuous tuning WGMs resonator based on an RAB microstructure. By changing the tensile strain applied to the RAB resonator, the WGMs resonance can be tuned by a PZT at a rate of $31.96 \mathrm{pm} / \mathrm{V}$ (strain-tuning rate $\sim 14.12 \mathrm{pm} / \mu \varepsilon$ ) with a potential tuning bandwidth of $14.12 \mathrm{~nm}$, which is over two times greater than its azimuthal FSR. Furthermore, a finite element model of the resonator was created for calculating the stress and deformation distribution under varying applied tensile strain, and the calculated results have finely explained the reasons of strain tuning. This fully tunable $\mathrm{RAB}$ resonator has unique advantages of a small mode volume, high quality factor exceeding $10^{6}$ and near-lossless WGMs.
Funding. This work was supported by National Natural Science Foundation of China (grant nos. 61425007, 61635007 and 61405125), Guangdong Natural Science Foundation (grant nos. 2015B010105007, 2014A030308007 and 2015A030313541), Science and Technology Innovation Commission of Shenzhen (grant nos. JCYJ20160427104925452, JCYJ20170412105604705 and JCYJ20170818093743767), Development and Reform Commission of Shenzhen Municipality foundation, and the Innovation-Leader Talent Project of Chongqing Science and Technology (CSTCCXLJR20171160).

\section{References}

[1] K. J. Vahala, Nature.424, 839(2003)

[2] V. Bulovic, V.G. Kozlov, V.B. Khalfin, and S.R. Forrest, Science. 279, 553(1998)

[3] X. Jiang, C. Zou, L. Wang, Q. Gong and Y. Xiao, Laser Photon. Rev $10,40(2016)$

[4] L. He, Ş. K. Özdemir and L. Yang, Laser Photon. Rev. 7, 60(2013)

[5] X. Fan, I. M. White, S. I. Shopova, H. Zhu, J. D. Suter and Y. Sun, Anal. Chim. Acta. 620, 8(2008)

[6] L. He, Ş. K. Özdemir, J. Zhu, W. Kim and L. Yang, Nat. Nanotechnol. 6, 428(2011)

[7] S. Panich, K. A. Wilson, P. Nuttall, C. K. Wood, T. Albrecht and J. B. Edel, Anal. Chem. 86, 6299(2014)

[8] A. D. Boozer, A. Boca, R. Miller, T. E. Northup and H. J. Kimble, Phys. Rev. Lett. 98, 193601(2007)

[9] T.Wilk, S.C. Webster, A.Kuhn and G. Rempe,Science. 317, 488(2007)

[10] F. Vollmer and S. Arnold, Nat. Methods. 5, 591(2008)

[11] M. S. Luchansky, A. L. Washburn, T. A. Martin, M. Iqbal, L. C. Gunn and R. C. Bailey, Biosens. Bioelectron. 26, 1283(2010)

[12] M. Sumetsky, Y. Dulashko, and R.S. Windeler, Opt. Lett. 35, 898(2010)

[13] M. Pollinger, D. O'Shea, F. Warken and A. Rauschenbeutel, Phys. Rev. Lett. 103, 053901(2009)

[14] M. Sumetsky, Y. Dulashko and R. S. Windeler, Opt. Lett. 35, 1866(2010)

[15] R. Madugani, Y. Yang, V. H. Le, J. M. Ward and S. Nic Chormaic,. IEEE Photonics Technol. Lett.28, 1134(2016)

[16] R. Henze, T.Seifert, J. Ward and O.Benson, Opt. Lett. 36, 4536(2011)

[17] M. Humar, M. Ravnik, S. Pajk and I. Musevic, Nat. Photonics. 3, 595(2009)

[18] C. Yang, H. Zhang, B. Liu, S. Lin, Y. Li and H. Liu, Opt. Lett. 42, 2988(2017)

[19] M. Mur, J. A. Sofi, I. Kvasic, A. Mertelj, D. Lisjak, V. Niranjan, I. Musevic and S. Dhara, Opt. Express. 25, 1073(2017)

[20] R. Chen, V. D. Ta and H. Sun, ACS Photonics. 1, 11(2014)

[21] S. Liu, K. Yang and Y. Wang, Sci. Rep. 5, 1(2015)

[22] S. Liu, Y. Wang and C. Liao, Opt. Lett. 39, 2121(2014)

[23] Z. Zhou, C. Zou, Y. Chen, Z. Shen, G. Guo, and C. Dong, Opt. Express 25,4046 (2017).

[24] M. Ye, M. Shen, and X. Lin,. Opt. Express 23, 25846 (2015)

[25] F. Brennecke, T. Donner, S. Ritter, T. Bourdel, M. Kohl and T. Esslinger, Nature, 450, 268(2007) 
1. Vahala K J. Optical microcavities[J]. nature, 2003, 424(6950): 839.

2. Bulović V, Kozlov V G, Khalfin VB, et al. Transform-limited, narrow-linewidth lasing action in organic semiconductor microcavities[J]. Science, 1998, 279(5350): 553-555.

3. Jiang $X F$, Zou $C L$, Wang $L$, et al. Whispering-gallery microcavities with unidirectional laser emission[J]. Laser \& Photonics Reviews, 2016, 10(1): 40-61.

4. He L, Özdemir \$̧ K, Yang L. Whispering gallery microcavity lasers[J]. Laser \& Photonics Reviews, 2013, 7(1): 60-82.

5. Fan X, White I M, Shopova S I, et al. Sensitive optical biosensors for unlabeled targets: A review[J]. analytica chimica acta, 2008, 620(1-2): 8-26.

6. He L, Özdemir Ş K, Zhu J, et al. Detecting single viruses and nanoparticles using whispering gallery microlasers[J]. Nature nanotechnology, 2011, 6(7): 428.

7. Panich $\mathrm{S}$, Wilson $\mathrm{K} \mathrm{A}$, Nuttall $\mathrm{P}$, et al. Label-free $\mathrm{Pb}$ (II) whispering gallery mode sensing using self-assembled glutathione-modified gold nanoparticles on an optical microcavity[]]. Analytical chemistry, 2014, 86(13): 6299-6306.

8. Boozer A D, Boca A, Miller R, et al. Reversible state transfer between light and a single trapped atom[J]. Physical Review Letters, 2007, 98(19): 193601.

9. Wilk T, Webster S C, Kuhn A, et al. Single-atom single-photon quantum interface[J]. Science, 2007, 317(5837): 488-490.

10. Vollmer F, Arnold S. Whispering-gallery-mode biosensing: label-free detection down to single molecules[J]. Nature methods, 2008, 5(7): 591.

11. Luchansky M S, Washburn A L, Martin T A, et al. Characterization of the evanescent field profile and bound mass sensitivity of a label-free silicon photonic microring resonator biosensing platform[J]. Biosensors and Bioelectronics, 2010, 26(4): 1283-1291.

12. Sumetsky M, Dulashko Y, Windeler R S. Optical microbubble resonator[J]. Optics letters, 2010, 35(7): 898-900.

13. Pöllinger $M, O$ 'Shea $D$, Warken $F$, et al. Ultrahigh- $Q$ tunable whisperinggallery-mode microresonator[J]. Physical review letters, 2009, 103(5): 053901.

14. Sumetsky M, Dulashko Y, Windeler R S. Super free spectral range tunable optical microbubble resonator[J]. Optics letters, 2010, 35(11): 1866-1868.

15. Madugani $\mathrm{R}$, Yang $\mathrm{Y}$, Le $\mathrm{V} H$, et al. Linear laser tuning using a pressuresensitive microbubble resonator[J]. IEEE Photon. Technol. Lett, 2016, 28(10): 1134-1137.

16. Henze R, Seifert $T$, Ward J, et al. Tuning whispering gallery modes using internal aerostatic pressure[J]. Optics letters, 2011, 36(23): 4536-4538.

17. Humar M, Ravnik M, Pajk $S$, et al. Electrically tunable liquid crystal optical microresonators[J]. Nature Photonics, 2009, 3(10): 595.

18. Yang $\mathrm{C}$, Zhang $\mathrm{H}$, Liu B, et al. Electrically tunable whispering gallery mode microresonator based on a grapefruit-microstructured optical fiber infiltrated with nematic liquid crystals[]]. Optics letters, 2017, 42(15): 29882991.

19. Mur M, Sofi J A, Kvasić I, et al. Magnetic-field tuning of whispering gallery mode lasing from ferromagnetic nematic liquid crystal microdroplets[J]. Optics Express, 2017, 25(2): 1073-1083.

20. Chen $R$, Ta V D, Sun H. Bending-induced bidirectional tuning of whispering gallery mode lasing from flexible polymer fibers[J]. ACS Photonics, 2014, 1(1): 11-16.

21. Liu S, Yang K, Wang Y, et al. High-sensitivity strain sensor based on in-fiber rectangular air bubble[J]. Scientific reports, 2015, 5: 7624.

22. Liu $S$, Wang $Y$, Liao $C$, et al. High-sensitivity strain sensor based on in-fiber improved Fabry-Perot interferometer[J]. Optics letters, 2014, 39(7): 21212124.

23. Zhou Z H, Zou C L, Chen Y, et al. Broadband tuning of the optical and mechanical modes in hollow bottle-like microresonators[J]. Optics Express, 2017, 25(4): 4046-4053.

24. Ye $M Y$, Shen $M X$, Lin $X M$. Transmission spectra of sausage-like microresonators[J]. Optics express, 2015, 23(20): 25846-25853.
25. Brennecke F, Donner T, Ritter S, et al. Cavity QED with a Bose-Einstein condensate[J]. Nature, 2007, 450(7167): 268. 